\title{
Linoleic acid intake, plasma cholesterol and 10-year incidence of CHD in 20000 middle-aged men and women in the Netherlands
}

\author{
Janette de Goede ${ }^{1}$, Johanna M. Geleijnse ${ }^{1 *}$, Jolanda M. A. Boer ${ }^{2}$, Daan Kromhout ${ }^{1}$ \\ and W. M. Monique Verschuren ${ }^{3}$ \\ ${ }^{1}$ Division of Human Nutrition, Wageningen University, PO Box 8129, 6700 EV Wageningen, The Netherlands \\ ${ }^{2}$ Centre for Nutrition and Health, National Institute for Public Health and the Environment, PO Box 1, 3720 BA Bilthoven, \\ The Netherlands \\ ${ }^{3}$ Centre for Prevention and Health Services Research, National Institute for Public Health and the Environment, \\ PO Box 1, 3720 BA Bilthoven, The Netherlands
}

(Submitted 8 February 2011 - Final revision received 19 May 2011 - Accepted 16 June 2011 - First published online 5 August 2011)

\begin{abstract}
We studied the associations of a difference in linoleic acid or carbohydrate intake with plasma cholesterol levels and risk of CHD in a prospective cohort study in the Netherlands. Data on diet (FFQ) and plasma total and HDL-cholesterol were available at baseline (1993-7) of 20069 men and women, aged 20-65 years, who were initially free of CVD. Incidence of CHD was assessed through linkage with mortality and morbidity registers. During an average of 10 years of follow-up, 280 CHD events occurred. The intake of linoleic acid ranged from 3.6 to $8.0 \%$ of energy (en\%), whereas carbohydrate intake ranged from 47.6 to 42.5 en $\%$ across quintiles of linoleic acid intake. Linoleic acid intake was inversely associated with total cholesterol and HDL-cholesterol in women but not in men. Linoleic acid intake was not associated with the ratio of total to HDL-cholesterol. No association was observed between linoleic acid intake and CHD incidence, with hazard ratios varying between 0.83 and 1.00 (all $P>0.05$ ) compared to the bottom quintile. We conclude that a $4-5 \mathrm{en} \%$ difference in linoleic acid or carbohydrate intake did not translate into either a different ratio of total to HDL-cholesterol or a different CHD incidence.
\end{abstract}

Key words: Carbohydrates: Coronary disease: PUFA: Epidemiology: Cholesterol

Linoleic acid $(18: 2 n-6)$ is an essential PUFA, and the main fatty acid of the $n-6$ PUFA family. A linoleic acid intake of $2 \%$ of energy $(\mathrm{en} \%) / \mathrm{d}$ is enough to prevent deficiency $^{(1)}$. Worldwide, the daily intake ranges from $3 \mathrm{en} \%$ (India) to $10 \mathrm{en} \%$ (Israel) $^{(2,3)}$. In the Netherlands, the average linoleic acid intake is $5-6 \mathrm{en} \%$ (approximately $14 \mathrm{~g} / \mathrm{d})^{(4-6)}$. n-6 PUFA currently contribute $85-90 \%$ of total PUFA intake, largely due to a high consumption of linoleic acid-rich vegetable oils $^{(7)}$.

In 2009, the American Heart Association summarised the scientific evidence on the association between $n$-6 PUFA and CVD and advised the consumption of 5-10en $\%$ of $n-6$ PUFA per $\mathrm{d}^{(8)}$. n-6 PUFA have a favourable effect on LDL-cholesterol and the ratio of total to HDL-cholesterol ${ }^{(9)}$. The positive effects of PUFA on CHD are therefore thought to be mainly mediated by fatty acid-induced changes in serum lipid levels. The total to HDL-cholesterol ratio is improved by substituting PUFA for SFA, yet also by substituting PUFA for carbohydrates ${ }^{(10)}$. Furthermore, carbohydrates also increase TAG, which are an independent risk factor for $\mathrm{CHD}^{(11)}$.

A recent meta-analysis of published prospective cohort studies did not demonstrate a positive effect of a higher PUFA intake in exchange with an isoenergetic amount of SFA on $\mathrm{CHD}^{(12)}$. However, another meta-analysis based on individual data that specifically addressed the isoenergetic exchange of SFA and PUFA showed that the replacement of 5 en $\%$ from saturated fat by PUFA was significantly associated with a $13 \%$ lower risk for coronary events, whereas the replacement of SFA by carbohydrates showed a significant direct association with $\mathrm{CHD}^{(13)}$. It has been suggested that replacement of SFA by carbohydrates, mainly refined carbohydrates, may exacerbate atherogenic dyslipidaemia ${ }^{(14)}$.

The aim of the present study was to evaluate the intake of linoleic acid, as an isoenergetic substitute for carbohydrates, in relation to both plasma cholesterol levels and 10-year incidence of CHD in a population-based cohort of over 20000 adults in the Netherlands.

Abbreviations: en \%, \% of energy; HR, hazard ratio; MI, myocardial infarction; MORGEN, Monitoring Project on Chronic Disease Risk Factors; Q, quintile. 


\section{Methods \\ Design and study population}

The 'Monitoring Project on Risk Factors for Chronic Diseases' (MORGEN) study is a Dutch population-based cohort of 22654 men and women aged 20-65 years. MORGEN is a part of the European Prospective Investigation into Cancer and Nutrition study ${ }^{(15)}$. Baseline (1993-7) information on diet, plasma cholesterol levels, lifestyle and cardiovascular risk factors was collected and participants were followed up for CVD end points. The present study was conducted according to the guidelines laid down in the Declaration of Helsinki, and all procedures involving human subjects/patients were approved by the Medical Ethics Committee of TNO Prevention and Health (Leiden, The Netherlands). Written informed consent was obtained from all subjects.

For the present study, participants who did not provide informed consent for vital status follow-up ( $n$ 701) were excluded, as well as seventy-two participants without dietary information and ninety-seven participants with extreme energy intakes $(<2094$ or $>18844 \mathrm{~kJ}$ for women and $<3350$ or $>20938 \mathrm{~kJ}$ for men). Furthermore, participants with a history of myocardial infarction (MI) or stroke at baseline were excluded ( $n$ 442). We also excluded participants who reported the use of serum lipid-modifying agents ( $n$ 203) or antihypertensive drugs ( $n$ 887), and 180 participants with diabetes resulting in 20069 participants (8988 men and 11081 women).

\section{Dietary assessment}

The habitual diet was assessed with a validated self-administered 178-item FFQ covering the previous year ${ }^{(16,17)}$. The FFQ included foods that covered the intake of foods and nutrients relevant to chronic disease aetiology for at least $90 \%$ of the national mean intake. Participants indicated the consumption of main food groups in times per $\mathrm{d}$, per week, per month, per year or as never, combined with questions on the relative intakes of foods within food groups (seldom/ never, sometimes, often or mostly/always). Nutrient intakes were calculated with the Dutch food composition table of 1998. For individual fatty acids, we used the table of 2001 , because the values were more complete. Total energy intake was calculated as the sum of energy from fat, carbohydrates and protein.

The reproducibility (estimated by two repeated measurements) and the relative validity (intake assessed by the FFQ compared to intakes assessed by 12-monthly 24-h recalls) of the FFQ for food groups and some nutrients were assessed among 121 Dutch men and women ${ }^{(16,17)}$. The Spearman rank correlations for the reproducibility of the FFQ after 6 months for total energy intake were 0.90 for men and 0.80 for women. Rank correlations were 0.83 and 0.77 for fat, 0.86 and 0.75 for protein and 0.91 and 0.85 for carbohydrates, in men and women, respectively. The relative validity of the FFQ for total energy intake was 0.77 for men and 0.62 for women. Rank correlations were 0.74 and 0.63 for fat, 0.68 and 0.56 for protein and 0.75 and 0.69 for carbohydrates, in men and women, respectively.

\section{Plasma lipid measurements}

Total cholesterol and HDL-cholesterol were measured in nonfasting EDTA plasma at the Lipid Reference Laboratory of the Erasmus Medical Center, Rotterdam, using enzymic methods. Total cholesterol was measured using an enzymic method; HDL-cholesterol was determined in the supernatant after precipitation of apoB-containing lipoproteins with phosphotungstic acid $/ \mathrm{MgCl}_{2}$. Performance for enzymatic total and HDL-cholesterol measurements fulfilled the National Cholesterol Education Program recommendations throughout the study $\operatorname{period}^{(18)}$.

\section{Mortality and morbidity}

Vital status was checked through linkage with the national population register. Participants were followed up for the occurrence of CHD through linkage with Statistics Netherlands for cause-specific mortality. Information on non-fatal events was provided by the national hospital discharge register based on a validated probabilistic linkage method described in more detail elsewhere ${ }^{(19)}$. It has been shown that on the national level, data from the Dutch hospital discharge register can be uniquely matched to a single person for at least $88 \%$ of the hospital admissions ${ }^{(19)}$. Incident CHD included fatal CHD (I20-I25), fatal and non-fatal cardiac arrest (I46) and non-fatal MI (I21-I22) according to the International Classification of Diseases, 10th revision (WHO). For hospital admissions and for causes of death coded until 1 January 1996, corresponding International Classification of Diseases, 9th revision codes were used. Participants were followed up until death, incident CHD, date of loss-to-follow-up due to emigration out of the Netherlands ( $n$ 693) or 1 January 2006, whichever came first.

\section{Other baseline characteristics}

Body weight, height and blood pressure were measured by trained research nurses. Self-administered questionnaires were used to assess the presence of diabetes, MI and stroke at baseline, medication use, parental history of MI, educational level and cigarette smoking ${ }^{(20)}$. Alcohol intake (based on the FFQ) was calculated in glasses/d. Baseline physical activity was assessed with a validated questionnaire in $76 \%$ of the cohort who were enrolled between 1994 and $1997^{(21)}$. For this subset, we calculated whether participants were engaged in activities with a metabolic equivalent score $\geq 4$ (yes/no). Cycling (yes/no) and sports (yes/no) were previously shown to be significantly inversely related to CVD incidence in this population $^{(22)}$.

\section{Statistical analysis}

Participants' characteristics by quintiles of linoleic acid intake expressed as en $\%$ are presented as mean values and standard deviations, medians with interquartile ranges or percentages. Correlations between the energy-adjusted intakes of different types of fatty acids were assessed using the Spearman rank correlation test. 
Mean plasma levels of total and HDL-cholesterol and the ratio of total to HDL-cholesterol by quintiles of linoleic acid intake (en\%) were computed using general linear models. $P$ for trend values of plasma cholesterol levels were calculated based on the continuous distribution of linoleic acid intake. We used Cox proportional hazards models to estimate relative risks for the incidence of CHD across quintiles of linoleic acid intake at baseline. Hazard ratios (HR) with 95\% CI were obtained using the bottom quintile of linoleic acid intake as the reference category. The proportional hazards assumption was tested and not rejected based on Schoenfeld residuals and visual inspection. In model 1 , we adjusted for total energy intake $(\mathrm{kJ} / \mathrm{d})$, age and sex. In model 2 , we additionally adjusted for BMI $\left(\mathrm{kg} / \mathrm{m}^{2}\right)$, alcohol intake (glasses/d), current cigarette smoking, high educational level (completed higher vocational training or university) (yes/no) and parental history of premature CHD (MI of father before the age of 55 years or MI of mother before the age of 65 years) (yes/no). In model 3 , we added intakes of fibre (g/d), protein, SFA, cis-MUFA, transfat and PUFA other than linoleic acid (all in en\%). The estimated HR of the full model can be interpreted as an isoenergetic replacement of carbohydrates with linoleic acid.

We assessed the impact of adjustments for systolic blood pressure, the total to HDL-cholesterol ratio and physical activity. Effect modification was evaluated for age and sex by adding product terms to the models. All statistical analyses were performed with SAS (version 9.1; SAS Institute, Inc., Cary, NC, USA). Two-sided $P$-values $<0.05$ were considered statistically significant.

\section{Results}

\section{Population characteristics}

Participants were on average 41.5 (SD 11.1) years at baseline, and $45 \%$ were male. The average intake of linoleic acid was $13.9(\mathrm{SD} 5.9) \mathrm{g} / \mathrm{d}$ or 5.6 (SD 1.6) en\%. Linoleic acid comprised $79 \%$ of total PUFA intake. During 8-13 years of follow-up (median 10.5 years), 199 men and 81 women experienced a CHD event, of which $19 \%$ was a fatal event.

The main sources of linoleic acid intake were margarines (21\%), oils (13\%), bread (12\%), nuts (10\%), pork meat (10\%) and sauces (9\%). Mean linoleic acid intake more than doubled across quintiles (Q), from $3.6 \mathrm{en} \%$ in Q1 to $8.0 \mathrm{en} \%$ in Q5. SFA and trans-fatty acids did not differ across quintiles, and carbohydrate intake decreased from 47.6 to $42.5 \mathrm{en} \%$. Polysaccharides did not differ between quintiles, whereas mono- and disaccharides decreased from 25 to $19 \mathrm{en} \%$ (Table 1). The Spearman correlations with linoleic acid were 0.98 for total PUFA, 0.43 for total fat, 0.32 for cis-MUFA and -0.35 for mono- and disaccharides.

\section{Linoleic acid, plasma lipid levels and CHD}

We observed interaction of sex on the association between linoleic acid intake and cholesterol levels. $P$-values for interaction were $<0.0001$ for total cholesterol, 0.01 for HDLcholesterol and 0.37 for the ratio of total to HDL-cholesterol.
In women, linoleic acid intakes were inversely associated with plasma total cholesterol levels in the fully adjusted model, with a mean total cholesterol level of $5 \cdot 28 \mathrm{mmol} / 1$ $(2042 \mathrm{mg} / \mathrm{l})$ in Q1 and $5 \cdot 14 \mathrm{mmol} / 1$ (1988 mg/l) in Q5 ( $P$-trend $<0.0001)$. In men, however, linoleic acid intake was not associated with total cholesterol. Linoleic acid intakes were inversely associated with plasma HDL-cholesterol across quintiles, in both men and women, although this inverse association did not reach statistical significance in men.

Linoleic acid intake was inversely, but not significantly, associated with the ratio of total to HDL-cholesterol in women, with ratio values between 3.65 (Q1) and 3.59 (Q5), whereas a positive, yet also non-significant, association was observed in men, with ratios varying between 4.65 (Q1) and 4.73 (Q5; Table 2).

After adjustment for potential confounders, linoleic acid intake was not associated with incident CHD. HR varied between 0.83 and 1.00 (all $P>0.05$ ) compared with the bottom quintile of linoleic acid intake (Table 3). The HR of incident CHD for a 5\% higher energy intake of linoleic acid with a concurrent lower intake of carbohydrates was 1.01 (95\% CI 0.86, 1.19).

We observed no interaction of sex or age $(\leq 50$ or $>50$ years) and linoleic acid intake in relation to incident CHD (data not shown). HR (95\% CI) for incident CHD after additional inclusion of the ratio of total to HDL-cholesterol and systolic blood pressure in the multivariable models were 0.92 (95\% CI $0.62,1.36), 0.82$ (95\% CI $0.55,1.24), 1.00(95 \%$ CI $0.68,1.48), 0.86(95 \%$ CI $0.57,1.30)$ for Q2-Q5 compared with Q1, respectively. For the subgroup with information on physical activity ( $n$ 15 423), the full model with and without physical activity yielded similar results (data not shown). Models based on isoenergetic substitution with SFA did not differ from the models based on substitution with carbohydrates (data not shown).

\section{Discussion}

In this large Dutch population-based cohort, a higher linoleic acid and concurrent lower carbohydrate intake was inversely associated with total cholesterol and HDL-cholesterol in women, but not in men. Linoleic acid intake was neither related to the ratio of total to HDL-cholesterol nor to CHD incidence.

In the present population-based study, the intakes of saturated fat and trans-fat were similar across the quintiles of linoleic acid intake and only carbohydrates varied between high and low linoleic acid intake. The present data set therefore allowed the analysis of real differences in intake between participants instead of statistically modelling these differences. Another strength of the present study was the almost complete follow-up of mortality. Furthermore, the procedure of identification of non-fatal events was validated in $36 \%$ of the participants of the present study by comparison against the clinical registry of the Cardiology Department of the Maastricht University Hospital. This showed a relatively high sensitivity (84\%) and positive predictive value (97\%) 
Table 1. Baseline characteristics of 20069 Dutch men and women, aged 20-65 years, by quintiles (Q) of energy percentages of linoleic acid intake (Mean values and standard deviations, unless indicated)

\begin{tabular}{|c|c|c|c|c|c|c|c|c|c|c|}
\hline & \multicolumn{2}{|c|}{ Q1 } & \multicolumn{2}{|c|}{ Q2 } & \multicolumn{2}{|c|}{ Q3 } & \multicolumn{2}{|c|}{ Q4 } & \multicolumn{2}{|c|}{ Q5 } \\
\hline & Mean & SD & Mean & SD & Mean & $S \mathrm{SD}$ & Mean & SD & Mean & $\overline{S D}$ \\
\hline$n$ & \multicolumn{2}{|c|}{4013} & \multicolumn{2}{|c|}{4014} & \multicolumn{2}{|c|}{4014} & \multicolumn{2}{|c|}{4014} & \multicolumn{2}{|c|}{4014} \\
\hline Male sex (\%) & \multicolumn{2}{|c|}{45} & \multicolumn{2}{|c|}{42} & \multicolumn{2}{|c|}{43} & \multicolumn{2}{|c|}{46} & \multicolumn{2}{|c|}{48} \\
\hline Age (years) & $41 \cdot 6$ & 11.8 & $40 \cdot 5$ & $11 \cdot 2$ & $40 \cdot 7$ & $10 \cdot 9$ & $41 \cdot 2$ & $10 \cdot 7$ & $43 \cdot 3$ & $10 \cdot 6$ \\
\hline \multicolumn{11}{|l|}{ PUFA } \\
\hline$g / d$ & 11.9 & 4.0 & $15 \cdot 0$ & 4.4 & $17 \cdot 0$ & 5.0 & $19 \cdot 6$ & 5.8 & 23.9 & 7.6 \\
\hline en $\%$ & 4.9 & 0.6 & $6 \cdot 1$ & 0.4 & 6.9 & 0.4 & $7 \cdot 8$ & 0.5 & 9.6 & $1 \cdot 2$ \\
\hline \multicolumn{11}{|l|}{ Linoleic acid } \\
\hline$g / d$ & 8.8 & 3.0 & 11.4 & 3.4 & $13 \cdot 3$ & 3.9 & $15 \cdot 8$ & 4.7 & $20 \cdot 0$ & $6 \cdot 6$ \\
\hline en $\%$ & 3.6 & 0.5 & 4.6 & 0.2 & $5 \cdot 4$ & 0.2 & $6 \cdot 3$ & 0.3 & 8.0 & $1 \cdot 1$ \\
\hline \multicolumn{11}{|l|}{$\alpha$-Linolenic acid } \\
\hline$g / d$ & $1 \cdot 1$ & 0.4 & 1.3 & 0.5 & 1.4 & 0.5 & 1.6 & 0.6 & 1.7 & 0.7 \\
\hline en $\%$ & 0.4 & 0.1 & 0.5 & 0.1 & 0.6 & 0.1 & 0.6 & 0.2 & 0.7 & 0.2 \\
\hline \multicolumn{11}{|l|}{ Cis-MUFA } \\
\hline$g / d$ & $27 \cdot 8$ & 10.5 & $30 \cdot 3$ & $10 \cdot 5$ & $31 \cdot 2$ & $10 \cdot 8$ & $32 \cdot 7$ & 11.4 & 33.7 & $12 \cdot 4$ \\
\hline en $\%$ & 11.4 & $2 \cdot 1$ & $12 \cdot 3$ & 2.0 & 12.5 & $2 \cdot 1$ & $12 \cdot 9$ & $2 \cdot 1$ & 13.5 & $2 \cdot 3$ \\
\hline TFA & & & & & & & & & & \\
\hline$g / d$ & $3 \cdot 7$ & 1.9 & 3.9 & 1.9 & 3.9 & 1.8 & 3.9 & 1.9 & 3.7 & 1.8 \\
\hline en\% & 1.5 & 0.5 & 1.6 & 0.5 & 1.6 & 0.5 & 1.5 & 0.5 & 1.4 & 0.5 \\
\hline SFA & & & & & & & & & & \\
\hline$g / d$ & $36 \cdot 9$ & 14.5 & 37.5 & $13 \cdot 3$ & $37 \cdot 2$ & $13 \cdot 1$ & $38 \cdot 0$ & $13 \cdot 3$ & $36 \cdot 8$ & $13 \cdot 2$ \\
\hline en $\%$ & $15 \cdot 1$ & 3.0 & $15 \cdot 1$ & 2.5 & 14.9 & $2 \cdot 4$ & $15 \cdot 0$ & $2 \cdot 3$ & $14 \cdot 7$ & $2 \cdot 3$ \\
\hline $\mathrm{P}: \mathrm{S}$ ratio & & & & & & & & & & \\
\hline Cholesterol (mg/d) & 244.0 & $96 \cdot 8$ & $245 \cdot 8$ & $92 \cdot 1$ & $241 \cdot 8$ & $89 \cdot 9$ & 244.2 & $90 \cdot 2$ & $231 \cdot 3$ & $91 \cdot 0$ \\
\hline Total fat & & & & & & & & & & \\
\hline $\mathrm{g} / \mathrm{d}$ & $81 \cdot 2$ & $29 \cdot 7$ & $87 \cdot 4$ & $29 \cdot 0$ & 89.9 & $29 \cdot 6$ & $95 \cdot 0$ & 31.4 & $98 \cdot 7$ & 33.3 \\
\hline en $\%$ & $33 \cdot 3$ & $5 \cdot 2$ & $35 \cdot 3$ & 4.5 & $36 \cdot 1$ & 4.5 & 37.6 & 4.4 & 39.4 & 4.5 \\
\hline Total carbohydrate (en\%) & $47 \cdot 6$ & 5.5 & $46 \cdot 0$ & 4.8 & $45 \cdot 3$ & 4.8 & $44 \cdot 1$ & 4.7 & 42.5 & 4.8 \\
\hline Mono and disaccharides (en\%) & $25 \cdot 0$ & $6 \cdot 2$ & $22 \cdot 5$ & $5 \cdot 3$ & 21.6 & $5 \cdot 2$ & $20 \cdot 5$ & 51 & $19 \cdot 1$ & 4.9 \\
\hline Polysaccharides (en\%) & $22 \cdot 6$ & 4.7 & 23.4 & 4.3 & 23.6 & 4.2 & $23 \cdot 6$ & 4.3 & 23.4 & $4 \cdot 2$ \\
\hline Fibre $(g / d)$ & $23 \cdot 8$ & 7.4 & 24.4 & $7 \cdot 0$ & 24.8 & $7 \cdot 0$ & $25 \cdot 3$ & $7 \cdot 2$ & $25 \cdot 6$ & $7 \cdot 3$ \\
\hline Protein (en\%) & $16 \cdot 1$ & $2 \cdot 7$ & $15 \cdot 8$ & $2 \cdot 3$ & $15 \cdot 7$ & $2 \cdot 2$ & $15 \cdot 5$ & $2 \cdot 1$ & $15 \cdot 3$ & $2 \cdot 1$ \\
\hline Energy intake $(\mathrm{MJ} / \mathrm{d})^{*}$ & $9 \cdot 0$ & $2 \cdot 8$ & $9 \cdot 1$ & $2 \cdot 7$ & 9.2 & $2 \cdot 7$ & $9 \cdot 3$ & $2 \cdot 7$ & 9.2 & $2 \cdot 7$ \\
\hline BMI $\left(\mathrm{kg} / \mathrm{m}^{2}\right)$ & 24.9 & 3.8 & 24.9 & 3.9 & 24.9 & 3.8 & 24.8 & 3.7 & 24.9 & 3.9 \\
\hline Body weight (kg) & $74 \cdot 2$ & $13 \cdot 6$ & $74 \cdot 1$ & 13.3 & 74.4 & 13.2 & $74 \cdot 3$ & $13 \cdot 1$ & 74.5 & 13.8 \\
\hline Current smoking (\%) & & & & & & & & & & \\
\hline Alcohol consumption (glasses/d) $\dagger$ & 0.4 & & 0.6 & & 0.7 & & 0.7 & & 0.8 & \\
\hline Interquartile range & & & & & & & & & & \\
\hline Highly educated $(\%) \ddagger$ & & & & & & & & & & \\
\hline Physically active (\%)§ & & & & & & & & & & \\
\hline Sports & & & & & & & & & & \\
\hline Cycling & & & & & & & & & & \\
\hline Parental history of MI (\%) & & & & & & & & & & \\
\hline $\mathrm{SBP}(\mathrm{mmHg})$ & $120 \cdot 4$ & $15 \cdot 5$ & $119 \cdot 8$ & $15 \cdot 5$ & $119 \cdot 3$ & $15 \cdot 3$ & $119 \cdot 3$ & $15 \cdot 0$ & $120 \cdot 7$ & $16 \cdot 3$ \\
\hline $\mathrm{DBP}(\mathrm{mmHg})$ & $76 \cdot 4$ & $10 \cdot 3$ & $76 \cdot 0$ & $10 \cdot 4$ & $75 \cdot 8$ & $10 \cdot 2$ & $75 \cdot 8$ & $10 \cdot 2$ & $76 \cdot 4$ & $10 \cdot 7$ \\
\hline Plasma total cholesterol (mmol/l) & $5 \cdot 3$ & 1.0 & $5 \cdot 3$ & 1.0 & $5 \cdot 2$ & 1.0 & $5 \cdot 3$ & $1 \cdot 0$ & $5 \cdot 3$ & $1 \cdot 1$ \\
\hline Plasma HDL-cholesterol (mmol/l) & 1.4 & 0.4 & 1.4 & 0.4 & 1.4 & 0.4 & 1.4 & 0.4 & 1.4 & 0.4 \\
\hline Total cholesterol:HDL-cholesterol & $4 \cdot 1$ & 1.5 & $4 \cdot 1$ & 1.4 & $4 \cdot 1$ & 1.5 & $4 \cdot 1$ & 1.5 & $4 \cdot 2$ & 1.5 \\
\hline
\end{tabular}

en\%, \% of energy; TFA, trans-fatty acids; P:S, polyunsaturated:saturated ratio; MI, myocardial infarction; SBP, systolic blood pressure; DBP, diastolic blood pressure.

* Total energy excluding alcohol intake.

† Median with interquartile range.

‡ University or higher vocation training.

$\S$ Available for participants enrolled between 1994 and 1997 ( $n$ 15 423).

for $\mathrm{MI}^{(23)}$. In addition, we also had detailed information on many potential confounders.

The present study also had limitations. Misclassification of subjects for linoleic acid intake may have occurred. However, we excluded participants with a history of MI or stroke, and also participants who used cholesterol-lowering or blood pressure-lowering medication, because these may have changed their diet. We therefore consider potential misclassification at baseline to be random rather than dependent on disease outcome. Multiple simultaneous and partly opposite effects of diet and lifestyle on CHD incidence had to be taken into account, including correlated types of fatty acids, partly due to presence in the same foods. Like in any other epidemiological study on diet and CHD, this may also have affected results in the present study. Although we attempted to disentangle the various possible simultaneous effects with the present statistical models, it is impossible to completely rule out confounding.

In the present study, linoleic acid intake was not significantly associated with the plasma total to HDL-cholesterol 
Table 2. Adjusted cholesterol levels by quintiles $(Q)$ of energy percentages of linoleic acid intake in 20069 Dutch men and women* $\dagger$ (Mean values with their standard errors)

\begin{tabular}{|c|c|c|c|c|c|c|c|c|}
\hline & \multirow[b]{2}{*}{$n$} & \multirow[b]{2}{*}{$\begin{array}{c}\text { Median } \\
\text { intake (en\%) }\end{array}$} & \multicolumn{2}{|c|}{ Model $1 \ddagger$} & \multicolumn{2}{|c|}{ Model $2 \S$} & \multicolumn{2}{|c|}{ Model 3\| } \\
\hline & & & Mean & SE & Mean & SE & Mean & SE \\
\hline \multicolumn{9}{|l|}{ Men } \\
\hline \multicolumn{9}{|c|}{ Total cholesterol $(\mathrm{mmol} / \mathrm{l})$} \\
\hline Q1 & 1797 & $3 \cdot 7$ & $5 \cdot 27$ & 0.02 & $5 \cdot 26$ & 0.02 & 5.25 & 0.02 \\
\hline Q2 & 1798 & 4.7 & $5 \cdot 30$ & 0.02 & $5 \cdot 30$ & 0.02 & $5 \cdot 30$ & 0.02 \\
\hline Q3 & 1798 & 5.4 & $5 \cdot 26$ & 0.02 & $5 \cdot 26$ & 0.02 & $5 \cdot 26$ & 0.02 \\
\hline Q4 & 1798 & $6 \cdot 3$ & $5 \cdot 33$ & 0.02 & $5 \cdot 34$ & 0.02 & $5 \cdot 35$ & 0.02 \\
\hline Q5 & 1797 & $7 \cdot 8$ & $5 \cdot 27$ & 0.02 & $5 \cdot 28$ & 0.02 & 5.29 & 0.02 \\
\hline$P$ trend & & & \multicolumn{2}{|c|}{0.90} & \multicolumn{2}{|c|}{0.49} & \multicolumn{2}{|c|}{0.31} \\
\hline \multicolumn{3}{|c|}{ HDL-cholesterol (mmol/l) } & & & & & & \\
\hline Q1 & 1797 & 3.7 & $1 \cdot 19$ & 0.01 & $1 \cdot 20$ & 0.01 & $1 \cdot 21$ & 0.01 \\
\hline Q2 & 1798 & 4.7 & $1 \cdot 20$ & 0.01 & $1 \cdot 20$ & 0.01 & $1 \cdot 20$ & 0.01 \\
\hline Q3 & 1798 & 5.4 & $1 \cdot 18$ & 0.01 & $1 \cdot 18$ & 0.01 & 1.18 & 0.01 \\
\hline Q4 & 1798 & $6 \cdot 3$ & $1 \cdot 19$ & 0.01 & $1 \cdot 19$ & 0.01 & $1 \cdot 18$ & 0.01 \\
\hline Q5 & 1797 & $7 \cdot 8$ & 1.20 & 0.01 & $1 \cdot 20$ & 0.01 & $1 \cdot 19$ & 0.01 \\
\hline$P$ trend & & & \multicolumn{2}{|c|}{0.38} & \multicolumn{2}{|c|}{0.61} & \multicolumn{2}{|c|}{0.16} \\
\hline \multicolumn{9}{|c|}{ Total cholesterol:HDL-cholesterol } \\
\hline Q1 & 1797 & 3.7 & 4.72 & 0.04 & $4 \cdot 70$ & 0.03 & 4.65 & 0.04 \\
\hline Q2 & 1798 & 4.7 & 4.68 & 0.04 & 4.68 & 0.03 & 4.67 & 0.03 \\
\hline Q3 & 1798 & 5.4 & 4.73 & 0.04 & 4.72 & 0.03 & 4.73 & 0.03 \\
\hline Q4 & 1798 & $6 \cdot 3$ & 4.74 & 0.04 & 4.77 & 0.03 & 4.79 & 0.03 \\
\hline Q5 & 1797 & $7 \cdot 8$ & 4.69 & 0.04 & $4 \cdot 70$ & 0.03 & 4.73 & 0.04 \\
\hline$P$ trend & & & \multicolumn{2}{|c|}{0.68} & \multicolumn{2}{|c|}{0.78} & \multicolumn{2}{|c|}{0.06} \\
\hline \multicolumn{9}{|l|}{ Women } \\
\hline \multicolumn{9}{|c|}{ Total cholesterol (mmol/l) } \\
\hline Q1 & 2216 & 3.8 & $5 \cdot 28$ & 0.02 & $5 \cdot 28$ & 0.02 & $5 \cdot 28$ & 0.02 \\
\hline Q2 & 2216 & 4.6 & $5 \cdot 29$ & 0.02 & $5 \cdot 28$ & 0.02 & $5 \cdot 28$ & 0.02 \\
\hline Q3 & 2217 & $5 \cdot 3$ & $5 \cdot 21$ & 0.02 & $5 \cdot 21$ & 0.02 & $5 \cdot 21$ & 0.02 \\
\hline Q4 & 2216 & $6 \cdot 2$ & $5 \cdot 22$ & 0.02 & $5 \cdot 22$ & 0.02 & $5 \cdot 22$ & 0.02 \\
\hline Q5 & 2216 & $7 \cdot 7$ & $5 \cdot 13$ & 0.02 & $5 \cdot 14$ & 0.02 & $5 \cdot 14$ & 0.02 \\
\hline$P$ trend & & & \multicolumn{2}{|c|}{$<0.0001$} & \multicolumn{2}{|c|}{$<0.0001$} & & \\
\hline HDL-chol & & & & & & & & \\
\hline Q1 & 2216 & 3.8 & 1.52 & 0.01 & 1.52 & 0.01 & 1.53 & 0.01 \\
\hline Q2 & 2216 & 4.6 & 1.52 & 0.01 & 1.53 & 0.01 & 1.53 & 0.01 \\
\hline Q3 & 2217 & $5 \cdot 3$ & 1.52 & 0.01 & 1.52 & 0.01 & 1.52 & 0.01 \\
\hline Q4 & 2216 & $6 \cdot 2$ & 1.51 & 0.01 & 1.51 & 0.01 & 1.51 & 0.01 \\
\hline Q5 & 2216 & $7 \cdot 7$ & 1.52 & 0.01 & 1.52 & 0.01 & 1.51 & 0.01 \\
\hline$P$ trend & & & & & & & & \\
\hline Total cho & & & & & & & & \\
\hline Q1 & 2216 & 3.8 & 3.67 & 0.02 & 3.66 & 0.02 & 3.65 & 0.02 \\
\hline Q2 & 2216 & 4.6 & 3.66 & 0.02 & 3.65 & 0.02 & 3.64 & 0.02 \\
\hline Q3 & 2217 & 5.3 & 3.62 & 0.02 & 3.62 & 0.02 & 3.62 & 0.02 \\
\hline Q4 & 2216 & $6 \cdot 2$ & 3.65 & 0.02 & 3.65 & 0.02 & 3.66 & 0.02 \\
\hline Q5 & 2216 & $7 \cdot 7$ & 3.57 & 0.02 & 3.58 & 0.02 & 3.59 & 0.02 \\
\hline$P$ trend & & & & & & & & \\
\hline
\end{tabular}

en\%, \% of energy.

* Total cholesterol was missing for $n 113$ and HDL-cholesterol was missing for $n 123$.

† Stratified analysis was based on sex-specific quintiles of linoleic acid.

$\ddagger$ Means adjusted for age, sex and total energy intake. For total cholesterol: $n 8951$ in men and $n 11005$ in women. For HDL-cholesterol and the total: HDL-cholesterol ratio: $n 8948$ in men and $n 10998$ in women.

$\S$ Means additionally adjusted for smoking, BMI, educational level, parental history of myocardial infarction and alcohol intake. For total cholesterol: $n 8876$ in men and $n 10909$ in women. For HDL-cholesterol and the total: HDL-cholesterol ratio: $n 8875$ in men and $n 10902$ in women.

$\|$ Means additionally adjusted for intake of dietary fibre, protein, SFA, MUFA, trans-fatty acids, PUFA other than linoleic acid. For total cholesterol: $n$ 8876 in men and $n 10909$ in women. For HDL-cholesterol and the total: HDL-cholesterol ratio: $n 8875$ in men and $n 10902$ in women.

ratio. A meta-analysis of controlled dietary intervention studies showed that the replacement of 1 en $\%$ of carbohydrates by PUFA would result in a reduction of the ratio of total to HDL-cholesterol by 0.032 . In observational studies of adults between 40 and 59 years, each one unit lower total to HDL-cholesterol ratio was associated with a $44 \%$ lower risk of $\mathrm{CHD}^{(9)}$. In the population of the present study, derived from controlled dietary intervention studies, the predicted difference of the total to HDL ratio between highest and lowest quintiles of linoleic acid intake was $-0 \cdot 15^{(10)}$, which corresponds to an approximately $7 \%$ lower CHD incidence. However, such a modest difference is difficult to detect considering the errors in observational food intake data.

A linoleic acid intake ranging between 3.6 and 8.0 en\% was not significantly associated with incident CHD in the present study. These results are in line with those from the cohort 
Table 3. Associations of linoleic acid intake* with incident CHD in 20069 Dutch men and women

(Numbers, hazard ratios and $95 \%$ confidence intervals)

\begin{tabular}{|c|c|c|c|c|c|c|c|c|c|}
\hline & \multirow[b]{2}{*}{$n$} & \multirow[b]{2}{*}{$\begin{array}{c}\text { Median } \\
\text { intake (en\%) }\end{array}$} & \multirow[b]{2}{*}{ Cases $(n)$} & \multicolumn{2}{|c|}{ Model $1 \dagger$} & \multicolumn{2}{|c|}{ Model $2 \ddagger$} & \multicolumn{2}{|c|}{ Model 3§ } \\
\hline & & & & HR & $95 \% \mathrm{Cl}$ & $\mathrm{HR}$ & $95 \% \mathrm{Cl}$ & $\mathrm{HR}$ & $95 \% \mathrm{Cl}$ \\
\hline Q1 & 4013 & 3.7 & 61 & 1.0 (ref) & & 1.0 (ref) & & $1 \cdot 0$ (ref) & \\
\hline Q2 & 4014 & 4.7 & 50 & 0.95 & $0.65,1.38$ & 0.93 & $0.63,1.35$ & 0.90 & $0.61,1.33$ \\
\hline Q3 & 4014 & 5.4 & 46 & 0.86 & $0.59,1.26$ & 0.86 & $0.58,1.27$ & 0.83 & $0.56,1.24$ \\
\hline Q4 & 4014 & $6 \cdot 2$ & 61 & 1.03 & $0.72,1.47$ & 1.04 & $0.73,1.50$ & 1.00 & $0.68,1.47$ \\
\hline Q5 & 4014 & $7 \cdot 7$ & 62 & 0.91 & $0.64,1.29$ & 0.94 & $0.65,1.34$ & 0.90 & $0.60,1.36$ \\
\hline Per $2 \%$ & & & & 1.00 & $0.87,1.15$ & 1.02 & $0.89,1.17$ & 1.01 & $0.86,1.19$ \\
\hline
\end{tabular}

en\%, \% of energy; HR, hazard ratio; $Q$, quintile.

* Values are HR with $95 \% \mathrm{Cl}$ in quintiles (Q1-Q5) of linoleic acid intake, using Q1 as the reference category.

$\dagger$ Adjusted for age, sex and total energy intake ( $n$ 20069)

$\ddagger$ Additionally adjusted for smoking, BMl, educational level, parental history of myocardial infarction and alcohol intake ( $n$ 19896).

$\S$ Additionally adjusted for dietary fibre, protein, SFA, cis-MUFA, trans-fatty acids and PUFA other than linoleic acid ( $n$ 19896).

studies in Finnish men ${ }^{(24)}$, Danish men and women ${ }^{(25)}$ and American men ${ }^{(26)}$, which also used models of isoenergetic substitution of PUFA for carbohydrates. A similar model was used in the Nurses' Health Study. However, in that study with 80000 women (1766 events), PUFA intake, ranging from $4 \cdot 1$ to $7 \cdot 4$ en $\%$, was inversely associated with a $25(95 \%$ CI 8, 40)\% lower CHD incidence in Q5 compared to Q1 ${ }^{(27)}$. Observational studies in the USA or Western Europe mostly covered relatively small ranges of intake of 5 to $10 \mathrm{en} \%$. To find associations within this range will be complicated by measurement error of intake in single dietary assessments ${ }^{(28)}$. Therefore, in observational studies, differences in linoleic acid intake may not translate into the predicted, although modest, differences in cholesterol and CHD risk.

On the basis of eight randomised trials, it has been shown that an increase in PUFA intake of $5 \%$ of energy was significantly associated with a $10 \%$ lower risk of coronary events ${ }^{(29)}$. However, this effect size was estimated from large contrasts of PUFA intake (on average $5 v .15$ en\%) between the intervention and control groups. Additionally, the PUFA interventions were mostly a combination of both a higher intake of $n-6$ and $n-3$ PUFA, whereas in the present study, we adjusted for PUFA other than linoleic acid. A recent meta-analysis separated the trials in the meta-analysis of Mozaffarian et al. ${ }^{(29)}$ into those on n-6 PUFA only and those with combined n-6 and n-3 PUFA interventions. The authors ${ }^{(30)}$ concluded that there was no indication of benefit of n-6 PUFA, although this statement was based on only two trials. However, it is clear from the information presented in that paper that the effect of a 5 en $\%$ difference in $n-6$ PUFA intake is less than the $10 \%$ difference in CHD incidence calculated from the dietary interventions for total PUFA.

In conclusion, in this large population-based study in the Netherlands, a 4-5 en\% difference in linoleic acid or carbohydrate intake did not translate into either a different ratio of plasma total to HDL-cholesterol or a different CHD incidence.

\section{Acknowledgements}

This work was supported by an unrestricted grant from the Product Board for Margarine, Fats and Oils, Rijswijk,
The Netherlands to the Wageningen University. The Monitoring Project on Risk Factors for Chronic Diseases (MORGEN) study was financially supported by the Ministry of Health, Welfare and Sport of the Netherlands, the National Institute for Public Health and the Environment, Bilthoven, The Netherlands and the Europe Against Cancer Program of the European Union. J. M. G. has received unrestricted grants from the Alpro Foundation, Belgium and from the Product Board for Margarine, Fats and Oils. J. M. G. and D. K. have received research grants from the Netherlands Heart Foundation, the $\mathrm{NIH}$ and Unilever Research and Development, The Netherlands, for the Alpha Omega Trial on $n$-3 fatty acids and CVD. The other authors report no conflicts of interest. J. G., J. M. G., D. K. and W. M. M. V. designed the research; J. M. A. B. and W. M. M. V. provided the data; J. G. analysed data and performed statistical analyses; J. G. drafted the paper; J. G., J. M. G. and D. K. had primary responsibility for final content. All authors read and approved the final manuscript.

\section{References}

1. Food and Agriculture Organization/World Health Organization (2008) Joint FAO/WHO Expert Consultation. Interim Summary and Conclusions and Dietary Recommendations on Total Fat \& Fatty Acids. Geneva: FAO/WHO.

2. Elmadfa I \& Kornsteiner M (2009) Dietary fat intake - a global perspective. Ann Nutr Metab 54, Suppl. 1, 8-14.

3. Kark JD, Kaufmann NA, Binka F, et al. (2003) Adipose tissue $n$ - 6 fatty acids and acute myocardial infarction in a population consuming a diet high in polyunsaturated fatty acids. Am J Clin Nutr 77, 796-802.

4. Voorlichtingsbureau voor de Voeding (1993) Zo eet Nederland: Resultaten Van de Voedselconsumptiepeiling 1992 (Dutch National Food Consumption Survey of 1992). The Hague: Voorlichtingsbureau voor de Voeding.

5. Voedingscentrum (1998) Zo eet Nederland: Resultaten van de Voedselconsumptiepeiling 1997-1998. (Dutch National Food Consumption Survey of 1997-1998). The Hague: Voedingscentrum.

6. Elmadfa I \& Kornsteiner M (2009) Fats and fatty acid requirements for adults. Ann Nutr Metab 55, 56-75.

7. Meyer BJ, Mann NJ, Lewis JL, et al. (2003) Dietary intakes and food sources of omega- 6 and omega- 3 polyunsaturated fatty acids. Lipids 38, 391-398. 
8. Harris WS, Mozaffarian D, Rimm E, et al. (2009) Omega-6 fatty acids and risk for cardiovascular disease: a science advisory from the American Heart Association Nutrition Subcommittee of the Council on Nutrition, Physical Activity, and Metabolism; Council on Cardiovascular Nursing; and Council on Epidemiology and Prevention. Circulation 119, 902-907.

9. Lewington S, Whitlock G, Clarke R, et al. (2007) Blood cholesterol and vascular mortality by age, sex, and blood pressure: a meta-analysis of individual data from 61 prospective studies with 55,000 vascular deaths. Lancet 370, 1829-1839.

10. Mensink RP, Zock PL, Kester AD, et al. (2003) Effects of dietary fatty acids and carbohydrates on the ratio of serum total to HDL cholesterol and on serum lipids and apolipoproteins: a meta-analysis of 60 controlled trials. Am J Clin Nutr 77, 1146-1155.

11. Hokanson JE \& Austin MA (1996) Plasma triglyceride level is a risk factor for cardiovascular disease independent of high-density lipoprotein cholesterol level: a meta-analysis of population-based prospective studies. J Cardiovasc Risk 3, 213-219.

12. Siri-Tarino PW, Sun Q, Hu FB, et al. (2010) Meta-analysis of prospective cohort studies evaluating the association of saturated fat with cardiovascular disease. Am J Clin Nutr 91, 535-546.

13. Jakobsen MU, O'Reilly EJ, Heitmann BL, et al. (2009) Major types of dietary fat and risk of coronary heart disease: a pooled analysis of 11 cohort studies. Am J Clin Nutr 89, 1425-1432.

14. Siri-Tarino PW, Sun Q, Hu FB, et al. (2010) Saturated fat, carbohydrate, and cardiovascular disease. Am J Clin Nutr 91, 502-509.

15. Riboli E \& Kaaks R (1997) The EPIC Project: rationale and study design. European Prospective Investigation into Cancer and Nutrition. Int J Epidemiol 26, Suppl. 1, S6-S14.

16. Ocké MC, Bueno-de-Mesquita HB, Goddijn HE, et al. (1997) The Dutch EPIC food frequency questionnaire. I. Description of the questionnaire, and relative validity and reproducibility for food groups. Int J Epidemiol 26, Suppl. 1, S37-S48.

17. Ocké MC, Bueno-de-Mesquita HB, Pols MA, et al. (1997) The Dutch EPIC food frequency questionnaire. II. Relative validity and reproducibility for nutrients. Int $J$ Epidemiol 26, Suppl. 1, S49-S58.

18. Houterman S, Verschuren WM, Oomen CM, et al. (2001) Trends in total and high density lipoprotein cholesterol and their determinants in The Netherlands between 1993 and 1997. Int J Epidemiol 30, 1063-1070.
19. De Bruin A, De Bruin EI, Gast A, et al. (2003) Linking Data of National Ambulant Register and GBA Data: Methods, Results and Quality Research (in Dutch). Koppeling van LMR- en GBA gegevens: methode, resultaten en kwaliteitsonderzoek. Voorburg/Heerlen: Statistics Netherlands.

20. Verschuren WM, Blokstra A, Picavet HS, et al. (2008) Cohort profile: the Doetinchem Cohort Study. Int J Epidemiol 37, $1236-1241$.

21. Pols M, Peeters P, Ocké M, et al. (1997) Estimation of reproducibility and relative validity of the questions included in the EPIC Physical Activity Questionnaire. Int J Epidemiol 26, 181S-189S

22. Hoevenaar-Blom MP, Wanda Wendel-Vos GC, Spijkerman AM, et al. (2010) Cycling and sports, but not walking, are associated with 10-year cardiovascular disease incidence: the MORGEN Study. Eur J Cardiovasc Prev Rehabil 18, 41-47.

23. Merry AH, Boer JM, Schouten LJ, et al. (2009) Validity of coronary heart diseases and heart failure based on hospital discharge and mortality data in the Netherlands using the cardiovascular registry Maastricht cohort study. Eur J Epidemiol 24, 237-247.

24. Pietinen P, Ascherio A, Korhonen P, et al. (1997) Intake of fatty acids and risk of coronary heart disease in a cohort of Finnish men. The Alpha-Tocopherol, Beta-Carotene Cancer Prevention Study. Am J Epidemiol 145, 876-887.

25. Jakobsen MU, Overvad K, Dyerberg J, et al. (2004) Dietary fat and risk of coronary heart disease: possible effect modification by gender and age. Am J Epidemiol 160, 141-149.

26. Mozaffarian D, Ascherio A, Hu FB, et al. (2005) Interplay between different polyunsaturated fatty acids and risk of coronary heart disease in men. Circulation 111, 157-164.

27. Oh K, Hu FB, Manson JE, et al. (2005) Dietary fat intake and risk of coronary heart disease in women: 20 years of follow-up of the nurses' health study. Am J Epidemiol 161, 672-679.

28. Jacobs DR Jr, Anderson JT \& Blackburn H (1979) Diet and serum cholesterol: do zero correlations negate the relationship? Am J Epidemiol 110, 77-87.

29. Mozaffarian D, Micha R \& Wallace S (2010) Effects on coronary heart disease of increasing polyunsaturated fat in place of saturated fat: a systematic review and meta-analysis of randomized controlled trials. PLoS Med 7, e1000252.

30. Ramsden CE, Hibbeln JR, Majchrzak SF, et al. (2010) n-6 Fatty acid-specific and mixed polyunsaturate dietary interventions have different effects on CHD risk: a meta-analysis of randomised controlled trials. Br J Nutr 104, 1586-1600. 